\section{SYNTHESIS OF PENEMS AND THEIR ANTIBACTERIAL ACTIVITIES}

Sir:

WOODWARD and his coworkers ${ }^{1)}$ were the first to synthesize penems (1) possessing thiazoline rings fused to $\beta$-lactams by using an intramolecular WitTig reaction of 4-acylthioazetidinylphosphoranes (2) at the ring formation stage. Meanwhile, the structure of thienamycin, a $\beta$-lactam antibiotic of high antibacterial potency and a wide spectrum, has recently been disclosed as that of a 1-carbadethiapenem (3) ${ }^{2}$, making the preparation of 2-(alkylthio)-penems even more attractive. Prompted by a recent report of a new synthetic approach to penems ${ }^{3,4}$, we describe here the result of our studies on 2-alkylthio-substituted penems.

Sodium n-propyl trithiocarbonate, prepared by addition of carbon disulfide to a methanolic sodium $n$-propylmercaptide solution, was treated with a stoichiometric amount of 4-acetoxyazetidin-2-one $\mathrm{e}^{5)}$ at room temperature to give the azetidinone trithiocarbonate* (4a) as fine yellow needles, mp $79 \sim 80^{\circ} \mathrm{C}(74 \%$ yield): IR (Nujol) $3210,1759 \mathrm{~cm}^{-1}$; NMR $\delta\left(\mathrm{CDCl}_{3}+\mathrm{D}_{2} \mathrm{O}\right) 1.04$ $(3 \mathrm{H}, \mathrm{t}, \mathrm{J}=7 \mathrm{~Hz}), \sim 1.8(2 \mathrm{H}, \mathrm{m}), 3.05(1 \mathrm{H}, \mathrm{dd}$, $\mathbf{J}=15.5,2.5 \mathrm{~Hz}), 3.55(1 \mathrm{H}, \mathrm{dd}, \mathbf{J}=15.5,5 \mathrm{~Hz})$, $3.42(2 \mathrm{H}, \mathrm{t}, \mathrm{J}=7 \mathrm{~Hz}), 5.66(1 \mathrm{H}, \mathrm{dd}, \mathrm{J}=5,2.5 \mathrm{~Hz})$. The azetidinone (4a) thus obtained was refluxed with $p$-nitrobenzyl glyoxylate in benzene to give the hemiaminal (4b) as a 1:1 epimeric mixture $(75 \%$ yield) whose treatment with thionyl chloride in the presence of 2,6-lutidine followed by reaction with triphenylphosphine afforded the phosphorane (4c) as yellow prisms, mp $200 \sim$ $202^{\circ} \mathrm{C}(50 \%$ yield): IR (Nujol) $1759,1657 \mathrm{~cm}^{-1}$. Heating of the phosphorane (4c) in xylene at $130 \sim 135^{\circ}$ for 14 hours gave 2-(propylthio) penem-3-carboxylate (5a) as fine needles, $\mathrm{mp}$ $150 \sim 151^{\circ} \mathrm{C} \quad(57 \%$ yield $):$ IR (Nujol) 1790, $1685 \mathrm{~cm}^{-1}$; UV $\lambda_{\max }$ (ethanol) $262.2 \mathrm{~nm}(\epsilon 16480)$, $339.5 \mathrm{~nm}$ ( $\epsilon$ 11460); NMR $\delta$

* All new compounds gave satisfactory elemental analyses.

$\left(\mathrm{CDCl}_{3}\right) 1.05(3 \mathrm{H}, \mathrm{t}, \mathrm{J}=6.5 \mathrm{~Hz}), \sim 1.8(2 \mathrm{H}, \mathrm{m})$, $2.98(2 \mathrm{H}, \mathrm{t}, \mathrm{J}=7 \mathrm{~Hz}), 3.51(1 \mathrm{H}, \mathrm{dd}, \mathrm{J}=16,2 \mathrm{~Hz})$, $3.90(1 \mathrm{H}, \mathrm{dd}, \mathrm{J}=16,3.5 \mathrm{~Hz}), 5.26(1 \mathrm{H}, \mathrm{d}, \mathrm{J}=14$ $\mathrm{Hz}), 5.56(1 \mathrm{H}, \mathrm{d}, \mathrm{J}=14 \mathrm{~Hz}), 5.77(1 \mathrm{H}, \mathrm{dd}, \mathrm{J}=3.5$, $2 \mathrm{~Hz}), 7.71(2 \mathrm{H}, \mathrm{d}), 8.32(2 \mathrm{H}, \mathrm{d})$. The carboxylic acid (5b) was obtained as its sodium salt (powder) by shaking 5a in hydrogen atmosphere over $10 \%$ palladium on charcoal in a buffer solution ( $\mathrm{pH} 7$ ) and was submitted to biological test.

Other 2-(alkylthio)penems as shown in Table 1 were also obtained from the corresponding mercaptans via analogous sequences. Penems possessing hydroxyl, amino or carboxylic acid groups in the side chain were obtained by starting from suitably protected mercaptans and by deprotection at a later stage of synthesis. Details will be reported in a forthcoming paper.

According to preliminary assays shown in Table 1, these 2-(alkylthio)penems have remarkable antibacterial activity against Gram-positive bacteria, resembling penicillins rather than cephalosporins. Further, these compounds are also active against some Gram-negative bacteria; however, they seem to show a weak activity against $\beta$-lactamase-producing bacteria. In general, introduction of hetero atoms in the side chain increased the activity of the penem molecule (No. 4, 5, 6, 8, 10, 11 and 16 19). Activity is also enhanced by the presence of more

Chart 1.
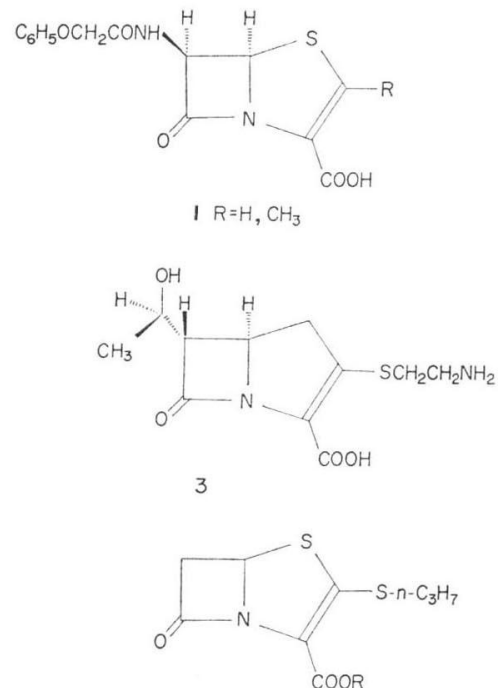

5a $R=P N B$ $5 b \quad R=H$

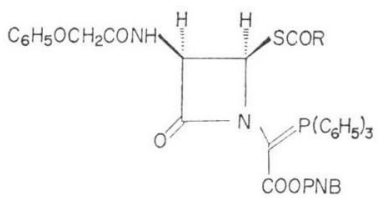

$2 \mathrm{R}=\mathrm{H}, \mathrm{CH}_{3}$

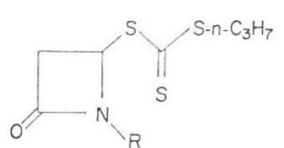

4a $\quad R=H$

$\mathrm{OH}$

4b $\mathrm{R}=\stackrel{\mathrm{C}}{\mathrm{C}} \mathrm{HCOOPNB}$ $\mathrm{P}\left(\mathrm{C}_{6} \mathrm{H}_{5}\right)_{3}$

4c $\mathrm{R}=$ CCOOPNB 
Table 1. Minimum inhibitory concentrations of 2-(alkylthio)penem-3-carboxylic acids.

\begin{tabular}{|c|c|c|c|c|c|c|c|c|c|c|c|}
\hline No. & 2-Substituent & $S \cdot a(\mathrm{~S})^{\mathrm{a}}$ & S. $a(\mathrm{R})^{\mathrm{b}}$ & $E . c(\mathrm{~S})^{\mathrm{c}}$ & $E \cdot c(\mathrm{R})^{\mathrm{d}}$ & S. $f^{\mathrm{e}}$ & $P . a^{\mathrm{f}}$ & $K \cdot p^{g}$ & $K . i^{\mathrm{h}}$ & $P . v^{\mathrm{i}}$ & $S . e^{\mathrm{j}}$ \\
\hline 1 & $-\mathrm{SCH}_{3}$ & 0.4 & 0.4 & 3.1 & 50 & 3.1 & $>200$ & 3.1 & 3.1 & 12.5 & 3.1 \\
\hline 2 & $-\mathrm{SCH}_{2} \mathrm{CH}_{2} \mathrm{CH}_{3}$ & 0.4 & 1.5 & 1.5 & $>100$ & 1.5 & $>100$ & 6.2 & 6.2 & 12.5 & 1.5 \\
\hline 3 & $-\mathrm{SCH}_{2} \mathrm{C}_{6} \mathrm{H}_{5}$ & $\leq 0.1$ & 0.2 & 12.5 & $>200$ & 3.1 & $>200$ & 25 & 25 & 6.2 & 6.2 \\
\hline 4 & $-\mathrm{SCH}_{2} \mathrm{CH}_{2} \mathrm{OH}$ & 0.4 & 1.5 & 0.8 & 100 & 0.8 & $>100$ & 0.8 & 0.8 & 6.2 & 0.8 \\
\hline 5 & $-\mathrm{SCH}_{2} \mathrm{CH}_{2} \mathrm{CH}_{2} \mathrm{OH}$ & 0.2 & 0.8 & 0.8 & 100 & 0.8 & $>100$ & 1.5 & 1.5 & 6.2 & 0.8 \\
\hline 6 & $-\mathrm{SCH}_{2} \mathrm{CH}_{2} \mathrm{OCOCH}_{3}$ & $\leq 0.1$ & 0.8 & 0.8 & $>50$ & 0.8 & $>50$ & 3.1 & 3.1 & 6.2 & 0.4 \\
\hline 7 & $-\mathrm{SCH}_{2} \mathrm{CH}_{2} \mathrm{CH}_{2} \mathrm{OCOCH}_{3}$ & 0.2 & 1.5 & 3.1 & $>100$ & 1.5 & $>100$ & 12.5 & 25 & 25 & 1.5 \\
\hline 8 & $-\mathrm{SCH}_{2} \mathrm{CH}_{2} \mathrm{OSO}_{2} \mathrm{CH}_{3}$ & 0.1 & 0.4 & 0.4 & $>200$ & 0.8 & $>200$ & 3.1 & 3.1 & 25 & 0.8 \\
\hline 9 & $-\mathrm{SCH}_{2} \mathrm{CH}_{2} \mathrm{OCOCOOCH}_{3}$ & 0.4 & 1.5 & 0.4 & $>200$ & 0.4 & $>200$ & 1.5 & 0.8 & 6.2 & 0.4 \\
\hline 10 & $-\mathrm{SCH}_{2} \mathrm{CH}_{2} \mathrm{OCH}_{2} \mathrm{CH}_{3}$ & 0.2 & 0.8 & 0.4 & 200 & 0.4 & $>200$ & 1.5 & 6.2 & 6.2 & 0.4 \\
\hline 11 & $-\mathrm{SCH}_{2} \mathrm{CH}_{2} \mathrm{SCH}_{3}$ & 0.2 & 0.8 & 0.4 & 200 & 0.4 & $>200$ & 1.5 & 3.1 & 12.5 & 0.4 \\
\hline 12 & $-\mathrm{SCH}_{2} \mathrm{CH}_{2} \mathrm{SCSN}_{-}^{\prime}$ & 0.05 & 0.4 & $>100$ & $>100$ & 100 & $>100$ & $>100$ & 100 & 25 & 100 \\
\hline 13 & $-\mathrm{SCH}_{2} \mathrm{CH}_{2} \mathrm{SCSNH}_{2}$ & 0.1 & 0.4 & 3.1 & $>50$ & 1.5 & $>50$ & 6.2 & 25 & 25 & 1.5 \\
\hline 14 & $-\mathrm{SCH}_{2} \mathrm{CH}_{2} \mathrm{COOH}$ & 6.2 & 6.2 & 0.8 & $>100$ & 1.5 & $>100$ & 3.1 & 1.5 & 25 & 1.5 \\
\hline 15 & $-\mathrm{SCH}_{2} \mathrm{CH}_{2} \mathrm{COOC}_{2} \mathrm{H}_{5}$ & $\leq 0.1$ & 0.4 & 6.2 & $>200$ & 1.5 & $>200$ & 6.2 & 12.5 & 25 & 1.5 \\
\hline 16 & $-\mathrm{SCH}_{2} \mathrm{CH}_{2} \mathrm{~N}_{3}$ & $\leq 0.1$ & 0.8 & 0.4 & 100 & 0.4 & $>100$ & 1.5 & 3.1 & 6.2 & 0.4 \\
\hline 17 & $-\mathrm{SCH}_{2} \mathrm{CH}_{2} \mathrm{NH}_{2}$ & 0.2 & 0.8 & 1.5 & 50 & 1.5 & 100 & 3.1 & 3.1 & 6.2 & 1.5 \\
\hline 18 & $-\mathrm{SCH}_{2} \mathrm{CH}_{2} \mathrm{CH}_{2} \mathrm{NH}_{2}$ & 0.1 & 0.8 & 1.5 & 50 & 1.5 & 50 & 3.1 & 3.1 & 12.5 & 1.5 \\
\hline 19 & $-\mathrm{SCH}_{2} \mathrm{CH}_{2} \mathrm{NHCOCH}_{3}$ & 0.8 & 3.1 & 0.8 & $>100$ & 1.5 & $>100$ & 3.1 & 1.5 & 25 & 1.5 \\
\hline 20 & $-\mathrm{SCH}_{2} \mathrm{CH}_{2} \mathrm{CH}_{2} \mathrm{NHCOCH}_{3}$ & 0.8 & 3.1 & 1.5 & $>100$ & 1.5 & $>100$ & 6.2 & 6.2 & 25 & 3.1 \\
\hline 21 & $-\mathrm{SCH}_{2} \mathrm{CH}_{2} \mathrm{NHCOCH}_{2} \mathrm{C}_{6} \mathrm{H}_{5}$ & 0.4 & 1.5 & 1.5 & $>100$ & 0.8 & $>100$ & 6.2 & 50 & 6.2 & 1.5 \\
\hline 22 & $-\mathrm{SCH}_{2} \mathrm{CH}_{2} \mathrm{NHCOCHC}_{6} \mathrm{H}_{4}-p-\mathrm{OH}$ & 0.8 & 3.1 & 1.5 & $>100$ & 1.5 & $>100$ & 3.1 & 12.5 & 25 & 3.1 \\
\hline 23 & $-\mathrm{SCH}_{2} \mathrm{CH}_{2} \mathrm{NHCON}^{\prime} \mathrm{CO}-\mathrm{CO}^{\prime} \mathrm{NC}_{2} \mathrm{H}_{5}$ & 1.5 & 6.2 & 6.2 & $>100$ & 6.2 & $>100$ & 25 & 100 & 50 & 12.5 \\
\hline \multirow[t]{3}{*}{24} & $-\mathrm{SCH}_{2} \mathrm{CH}_{2} \mathrm{NHCOC}\left(=\mathrm{NOCH}_{3}\right) \mathrm{CH}_{3}$ & 0.8 & 3.1 & 6.2 & $>100$ & 6.2 & $>100$ & 25 & 50 & 50 & 12.5 \\
\hline & Ampicillin & $\leq 0.1$ & 1.5 & 3.1 & $>200$ & 1.5 & $>200$ & 50 & 6.2 & 1.5 & 0.4 \\
\hline & Cephalexin & 0.8 & 3.1 & 6.2 & 6.2 & 12.5 & $>200$ & 6.2 & 200 & 12.5 & 3.1 \\
\hline
\end{tabular}

M.I.C. values are in $\mathrm{mcg} / \mathrm{ml}$, and were determined in heart infusion agar.

a) Staphylococcus aureus FDA 209P JC. b) Staphylococcus aureus 56 (PCase $\left.{ }^{+}\right)$. c) Escherichia coli NIHJ JC-2. d) Escherichia coli 609 (CSase ${ }^{+}$).

e) Shigella flexneri IID 642. f) Pseudomonas aeruginosa 1001. g) Klebsiella pneumoniae 806. h) Klebsiella sp. 846. i) Proteus vulgaris 1430.

j) Salmonella enteritidis $\mathrm{G}$. 
polar functions; for instance, the hydroxy group (No. 4 and 5) as compared to the acetoxy group (No. 6 and 7). Although most of these penems show no anti-Pseudomonas activity, penems possessing the amino function (No. 17 and 18) exhibit significant activity against $P$ seudomonas aeruginosa, suggesting a correlation between the cysteaminyl side chain of the thienamycin (3) and its excellent anti-Pseudomonas activity. Introduction of a bulky alkyl or acylamino groups or an ester function reduces activity against some Gram-negative bacteria (No. 3, 12,15 and $21 \sim 24$ ).

These penems were found to be unstable under long storage and their bioavailability seemed rather low considering that the urinary recovery of the penem No. 10 parenterally administrated to mice was only $5.8 \%$.

\section{SADAO OidA \\ AKIRA YOSHIDA \\ TERUo Hayashi \\ NORIKO TAKEDA \\ TAKUzo Nishimura \\ EIJI OHKI}

Central Research Laboratories, Sankyo Co., Ltd., Hiromachi, Shinagawa-ku, Tokyo, 140, Japan

(Received November 12, 1979)

\section{References}

1) Ernest, I.; J. Gosteli, C. W. Greengrass, W. Holick, D. E. Jackman, H. R. Pfaendler \& R. B. WOODWARD: The penems, a new class of $\beta$-lactam antibiotics: 6-acylaminopenem-3carboxylic acid. J. Am. Chem. Soc. 100: $8214 \sim 8222,1978$

2) Albers-Schönberg, G.; B. H. Arison, O. D. Hensens, J. Hirshifield, K. Hoogsteen, E. A. KaczKa, R. E. Rhodes, J. S. Kahan, F. M. Kahan, R. W. Ratcliffe, E. Walton, L. J. Ruswinkle, R. B. Morin \& B. G. Christensen: Structure and absolute configuration of thienamycin. J. Am. Chem. Soc. 100: 6491 6499, 1978

3) Dininno, F.; E. V. Linek \& B. G. Christensen: Penems. 1. Penem formation via a novel oxidative addition process mediated by copper (I). J. Am. Chem. Soc. 101: 2210 2211, 1979 Oida, S.; A. Yoshida, T. Hayashi, E. NakaYAma, S. SATo \& E. OHKi: 7-Oxo-2-thia-1azabicyclo[3.2.0]hept-3-ene, a revised structure for penems synthesized via oxidative addition mediated by copper (I). Tetrahedron Lett. in press

4) Cherry, P. C.; C. E. Newall \& N. S. Watson: Synthesis of antibacterial pen-2-em-3-carboxylic acids from clavulanic acid. J. Chem. Soc., Chem. Comm. 1979: 663 664, 1979

5) Clauss, K.; D. Grim \& G. Rossel: $\beta$-Lactame mit über Heteroatome gebundenen Substituenten. Liebigs Ann. Chem. 1974: 539 560, 1974 